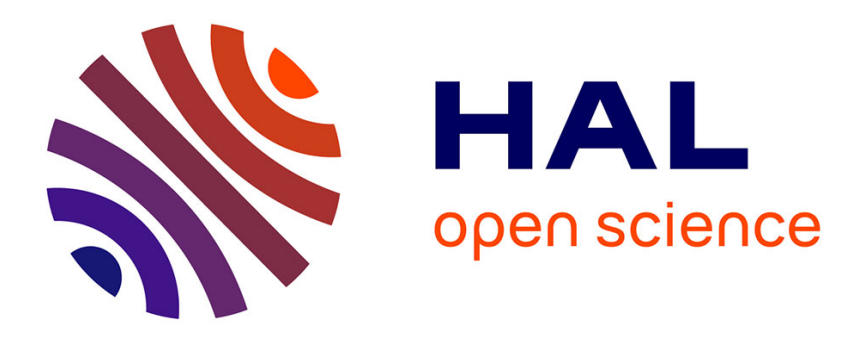

\title{
Vaches et blés sur le papier Socialisations à l'écrit du monde agricole
}

\author{
Nathalie Joly
}

\section{To cite this version:}

Nathalie Joly. Vaches et blés sur le papier Socialisations à l'écrit du monde agricole. Communication \& langages, 2009, Mars 2009 (159), pp.77-90. 10.4074/S0336150009001070 . hal-02656412

\section{HAL Id: hal-02656412 \\ https://hal.inrae.fr/hal-02656412}

Submitted on 29 May 2020

HAL is a multi-disciplinary open access archive for the deposit and dissemination of scientific research documents, whether they are published or not. The documents may come from teaching and research institutions in France or abroad, or from public or private research centers.
L'archive ouverte pluridisciplinaire HAL, est destinée au dépôt et à la diffusion de documents scientifiques de niveau recherche, publiés ou non, émanant des établissements d'enseignement et de recherche français ou étrangers, des laboratoires publics ou privés. 


\section{Vaches et blés sur le papier}

Socialisations à l'écrit du monde agricole
NATHALIE JOLY

« Ne vous laissez pas noyer sous la paperasse ! ». Ce titre d'un récent dossier de presse ${ }^{1}$ s'adressant à des éleveurs relance la réflexion sur les transformations de l'agriculture. Lorsque la vache folle envahit les écrans en 1996, les Français découvrent avec stupeur combien la réalité de l'élevage est en fait éloignée de leurs souvenirs d'enfance, de " ces bonnes vaches aux yeux si doux » dont l'image est exploitée par les publicitaires et les artistes ${ }^{2}$. La surprise est sensiblement équivalente (mais moins angoissante) quand un public non averti découvre le foisonnement d'écrits qui entoure aujourd'hui le travail agricole. Depuis que la traçabilité s'est imposée comme mode de gouvernement des risques ${ }^{3}$ et que la contractualisation règle une large part des relations que les agriculteurs entretiennent avec l'administration et leurs partenaires économiques, produire des bovins et des cultures exige de produire et de détenir quantité de documents : passeport des animaux, carnet sanitaire, ticket de pesée, plan d'épandage, carnet de pâturage... Cette activité « paperassière » ne constitue pas seulement une charge supplémentaire de travail. Elle inquiète les professionnels en ce qu'elle leur fait encourir un risque d'un genre nouveau, celui de ne pas être à jour dans leurs papiers et d'être pénalisés lors de contrôles. Après s'être fortement industrialisée, l'agriculture serait-elle en passe de se bureaucratiser?

1. Dans la revue Réussir Lait Elevage, $n^{\circ}$ 183, 2005.

2. André Micoud, "Ces bonnes vaches aux yeux si doux ", Communications, $\mathrm{n}^{\circ}$ 7, Paris, Seuil, 2003.

3. Didier Torny, "La traçabilité comme technique de gouvernement des hommes et des choses ", Politix, $\mathrm{n}^{\circ} 44,1998$.
En agriculture comme ailleurs, l'activité des professionnels apparaît de plus en plus équipée par toute sorte d'instruments destinés à noter, à enregistrer ou à mesurer. Pour cerner les enjeux et la nature exacte des changements signalés par ce constat, l'article propose de s'intéresser aux pratiques ordinaires de l'écrit dans le métier d'agriculteur et de les resituer dans une perspective historique. En décrivant plus particulièrement une pratique extrêmement répandue dans le monde agricole, la tenue d'agendas qui recueillent une chronique quotidienne des occupations sur la ferme, il éclaire par ce biais la diversité des modelages dont les écrits du travail ont pu faire l'objet de la part des institutions d'enseignement et de vulgarisation tout au long des $\mathrm{XIX}^{\mathrm{e}}$ et $\mathrm{XX}^{\mathrm{e}}$ siècles et dont le sens général est celui d'une adaptation à un ordre économique et technique et d'une rationalisation des méthodes de gestion. II met également en évidence les ressources cognitives associées à cette mise en mémoire du travail agricole, les réglages de l'activité qu'elle autorise, les formes de jugement et de réflexivité qu'elle soutient. C'est donc à une réflexion sur les modes d'appropriation des pratiques de l'écrit à laquelle l'auteur invite, nourrie d'exemples de détournements et de reformulations que les agriculteurs ont régulièrement opérés face aux cadrages normatifs qui leur étaient proposés. Une telle perspective s'avère particulièrement utile à la saisie des transformations en cours concernant les écrits du travail. Confrontés depuis quelques années à des exigences croissantes de traçabilité de leurs pratiques, les agriculteurs sont davantage sommés que par le passé de recourir à des canevas d'écriture standardisés. Se pose alors une série de questions sur les modalités de l'articulation entre des écrits confectionnés " pour soi " et des écrits destinés à " faire preuve " que l'auteur esquisse en conclusion, pour préciser des orientations de recherche à venir. 
Partant de cette question, nous invitons le lecteur à un détour par les pratiques ordinaires de l'écrit dans les exploitations. Si l'agriculture n'échappe pas à « la contrainte généralisée du compte rendu d'activité ${ }^{4}$, elle présente sous cet angle une histoire bien singulière et méconnue. Singulière, parce que l'écriture fait partie de longue date du quotidien de nombreux agriculteurs, du fait de multiples socialisations à l'écrit. Méconnue, en raison de stéréotypes - les historiens nous ont habitués à une paysannerie empreinte de traditions orales - mais aussi d'un certain malaise : alors qu'enseignants et agents de développement se sont appliqués à faire écrire les agriculteurs pour leur travail, ceux-ci ont en retour façonné leurs systèmes de notes à distance des modèles. Les pratiques ordinaires de l'écrit sont donc peu visibles, puisque déployées à l'ombre de la prescription. Le propos de cet article est précisément de leur donner visibilité et intelligibilité, au regard de ce fond historique et des évolutions en cours.

\section{L'OBJET-AGENDA}

L'environnement graphique d'une exploitation agricole apparaît aujourd'hui très complexe. Calendriers, carnets, cahiers, agendas, logiciels, ordinateurs de poche, et de manière plus insolite, panneaux d'armoires et portes de grange servent à l'inscription d'informations durables. Tableaux, bouts de carton, dos d'enveloppes, post it recueillent brouillons et notes éphémères. Par ailleurs, une masse impressionnante d'écrits imprimés (résultats du contrôle laitier, analyses de sol, presse professionnelle, formulaires de primes, fascicules commerciaux, factures...) afflue quotidiennement dans les fermes et s'accumule dans le bureau de l'agriculteur ${ }^{5}$, ou plus fréquemment, dans les buffets et tiroirs. Pour les besoins de l'activité, l'écriture se véhicule parfois, comme l'agenda glissé dans la cotte de travail, le carnet logé dans la boîte à outil du tracteur.

Parmi les supports qui sollicitent l'inscription, l'objet-agenda apparaît comme un vestige du passé. Une écriture, jamais relâchée, en noircit les pages suivant un mode d'emploi bien particulier : inscrire non pas "les choses à faire » comme l'usage le voudrait, mais « ce qui est fait chaque jour». Cette volonté d'engranger les événements de la journée rapproche le support d'un genre documentaire bien connu des historiens du XVIII ${ }^{\mathrm{e}}$ siècle, le livre de raison. Initialement construit autour des comptes et des événements familiaux, et tenu par des notables et des érudits provinciaux - dont agronomes et grands propriétaires -, le livre de raison restitue souvent le quotidien « dans son rythme, ses aspects matériels les plus prosaïques, ses activités les plus ordinaires $»^{6}$. Par l'économie de mots et la scansion événementielle qui le caractérise, il fixe un style aisé à reproduire, comme en témoignent les rares documents conservés qui ont appartenu à de

4. Sophie Pène, "Traces de mains sur les écrits gris ", dans Paroles au travail, J. Boutet, dir., Paris, l'Harmattan, 1995.

5. Terme utilisé ici par commodité, car ce sont souvent les conjointes d'exploitants qui gèrent les papiers. De plus, cette activité se trouve de plus en plus distribuée entre les membres des « collectifs de travail »- associés et salariés - qui composent aujourd'hui nombre exploitations.

6. Madeleine Foisil, "L'écriture du for privé " in : Histoire de la vie privée. De la Renaissance aux Lumières, Ariès P., Duby G. (dir.), Paris, Editions du Seuil, tome 3, p. 334, 1985, réed 1999. 
simples laboureurs ${ }^{7}$. Cette appropriation de l'écrit par la paysannerie va de pair avec le succès éditorial de " l'agenda-calepin » au cours du XIX ${ }^{\mathrm{e}}$ siècle. Dans sa recension des almanachs à travers les âges, John Grand-Carteret ${ }^{8}$ évoque la vulgarisation du support qu'on appelait le nécessaire : "le premier Empire, avec son esprit précis, méthodique, semble avoir exercé une influence décisive sur le développement de ce recueil annuel qui n'est, après tout, qu'un livre de comptes, quoique l'on ait déjà essayé et qu'on semble, à nouveau, vouloir essayer d'en faire une sorte de livre de raison, pour les notes et les pensées individuelles ». Avant qu'il ne devienne l'instrument banalisé que l'on sait, l'objet-agenda engage donc très tôt de " petits scripteurs ${ }^{9}$ à tenir la plume pour faire bon usage du temps.

\section{MOdÈLES D'ÉCRITURE ET PAYSANS MODÈLES}

Qu'un genre documentaire soit en vogue au XIX ${ }^{\mathrm{e}}$ siècle ne suffit pas à en assurer la diffusion. Pour qu'une large frange de la paysannerie se l'approprie, encore faut-il qu'elle en perçoive l'intérêt, et bien sûr, qu'elle possède des rudiments d'écriture. Deux leviers se trouvent réunis au cours de la seconde moitié du $\mathrm{XIX}^{\mathrm{e}}$ siècle pour favoriser de nouvelles postures de travail et l'apprentissage de techniques scripturales qui étaient jusqu'alors l'apanage de grands propriétaires : premièrement, un ensemble de découvertes scientifiques - utilisation des engrais, perfectionnement des instruments aratoires et progrès de la génétique - qui impose une sortie des " routines » et l'expérimentation de nouvelles techniques ; deuxièmement, une forte croissance des échanges commerciaux - due à l'amélioration des infrastructures routières et au développement des chemins de fer - qui stimule le développement de compétences gestionnaires et le suivi des transactions. Preuve d'un changement de mentalité, l'analphabétisme commence à être ressenti comme une indignité dans les campagnes et les paysans se tournent vers l'école ${ }^{10}$ pour acquérir des notions d'écriture, de calcul et de comptabilité. En écho à cette demande d'éducation (ou la devançant dans certains cas), un dispositif d'enseignement professionnel et de vulgarisation est mis sur pied, à l'initiative de l'État et d'institutions privées. Il s'agit d'instruire les jeunes se destinant à l'agriculture, mais également les paysans en exercice, grâce à des cours du soir, des conférences agricoles et la possibilité de suivre des études par correspondance. Un dispositif ambitieux de diffusion de connaissances donc, mais également

7. Pour un aperçu, se reporter à l'ouvrage de Jean Vassort, Les papiers d'un laboureur au siècle des Lumières. Pierre Bordier : une culture paysanne, Seyssel, Champ Vallon, 1999 et à celui de Michel Vernus, Une vie paysanne en Franche-Comté. Le livre de raison de Jean-Claude Mercier, laboureur à Mamirolle, 1740-1749, Vesoul, Editions de folklore comtois, 1999.

8. Les Almanachs français (1600-1895), Bibliographie-Iconographie, J. Alisié et Cie Editeurs, 1896, p. LXX.

9. Florence Weber propose ce terme par analogie à la notion statistique de "petits lecteurs ", dans "L'ethnographe et les scripteurs populaires. Quelques remarques sur l'écriture comme interaction et comme compétence ", Enquête, nº $8,1993$.

10. Dans sa monographie villageoise sur Mazières en Gâtine, Roger Thabault restitue finement les changements qui affectent les campagnes à cette période et leurs effets quant à la demande d'éducation de la paysannerie, dans Mon village : l'ascension d'un peuple, 1848-1914, Paris, Delagrave, 1945. 
d'encadrement des pratiques de l'écrit, ce que nous mettons en relief dans la partie suivante.

\section{Passer écriture}

Il faut remonter à l'expérience pionnière de l'agronome C. Mathieu de Dombasle pour comprendre la façon dont " travail agraire » et " écriture » vont se trouver étroitement imbriqués au long d'un siècle d'enseignement agricole. Ce dernier, réputé pour son invention de la charrue métallique, mais également pour ses réflexions pédagogiques, compare souvent la science agronomique à la médecine, toutes deux art d'interprétation. D'où son attachement à développer des qualités d'observation chez les futurs régisseurs qu'il se charge de former dans son Institut de Roville, créé en 1826 : "Il ne suffit pas de connaître une ferme en masse, il faut avoir étudié pendant longtemps, et tous les jours, chacun des champs qui la composent, les avoir observés dans toutes les saisons de l'année, dans toutes les circonstances de sécheresse et d'humidité, et couverts de plusieurs espèces de récoltes ${ }^{11}$. L'approche empirique qu'il préconise dépasse d'ailleurs le cadre pédagogique. Étant donné les lacunes des connaissances scientifiques à cette époque, l'agronome invite à une " pratique intellectuelle » de l'agriculture, c'està-dire une pratique fondée sur l'observation des faits et des circonstances dans lesquelles ils se produisent.

Compiler des observations engage une écriture soutenue. Si l'agronome enregistre lui-même quantité d'informations relatives à la gestion de son domaine et aux expérimentations pratiquées, à partir de supports qu'il confectionne et publie dans les Annales de Roville, il ne manque pas une occasion d'initier ses étudiants à la prise de note. Évoquant l'organisation de l'enseignement dispensé à son domaine, C. Mathieu de Dombasle explique par exemple que « chaque soir, les élèves assistent à l'Ordre où l'on passe écriture de toutes les opérations de la journée, sous la dictée des chefs qui les ont fait exécuter ${ }^{12}$, que c'est là une manière commode de donner ses ordres au personnel, et dans le même temps, de guider la formation des apprentis régisseurs. Ces derniers ont en effet pour consigne de puiser dans ce registre d'activités pour formuler leurs questionnements sur l'activité du domaine et s'en entretenir avec leur précepteur, lors de conférences hebdomadaires.

Le procédé fait école et lorsque les premiers établissements d'enseignement professionnel ouvrent leurs portes, après le décret du 3 octobre 1848, le législateur inscrit à l'emploi du temps une demi-heure destinée au " passage des écritures », allant jusqu'à conserver l'appellation de l'Ordre pour rendre justice à son instigateur. Puis, au fil des années, les canevas d'écriture se précisent, modelant la confection des chroniques du travail et leurs usages pédagogiques ${ }^{13}$. Une circulaire du

11. Christophe J.A. Mathieu de Dombasle, "Situation de l'établissement de Roville ", Annales de Roville, $4^{\mathrm{e}}$ livraison, 1828, Annales d'histoire des enseignements agricoles, 1987, ${ }^{\circ} 2$, 1987, pp. 73-74.

12. Op. cit., 1828, p. 28.

13. Pour une analyse plus détaillée, Nathalie Joly, "Chroniques du travail et livres de comptes : la diffusion de pratiques d'écriture en milieu paysan entre le XIX ${ }^{\mathrm{e}}$ et le $\mathrm{XX}^{\mathrm{e}}$ siècle ", dans Les enjeux de la formation des acteurs de l'agriculture, Dijon, Educagri Editions, 2000. 
25 octobre 1918 prévoit, par exemple, la tenue d'un agenda des travaux et d'un livre d'exploitations dans les établissements publics du second degré : «L'agenda de travaux est le livre où l'élève inscrit au jour le jour la nomenclature des travaux auxquels il a pris part et où il relève ses observations relatives à la culture, à l'étable et au jardin. Le livre d'exploitation est un recueil où l'élève, à l'aide de constatations personnelles et des renseignements techniques qu'il a pu se procurer, résume, chaque semaine, l'ensemble des faits relatifs à l'administration du domaine ». Un plan d'écriture sensiblement identique est proposé dans l'enseignement privé. Les paysans inscrits à des études par correspondance doivent tenir un «cahier de ferme » en respectant les consignes suivantes : "Sur la page de gauche, divisée en deux colonnes, les jeunes gens notent, chaque jour, ce qu'ils voient : une culture, une opération quelconque dans les champs, sera inscrite en quatre mots sur la première colonne ; la seconde recevra l'inscription des travaux accessoires, ce qui est plutôt travail de ferme que de culture. Ici, ils se contentent de lire sur le grand livre de la pratique, ils ne commentent pas. Mais sur la page de droite, une fois par semaine, en regard de cette chronique sèche, ils dégagent de leurs observations les leçons qui en ressortent ${ }^{14}$. Citons encore le «cahier d'exploitation » placé au centre de la pédagogie des Maisons Familiales Rurales et que les apprentis renseignent à l'aide d'un " plan d'étude ${ }^{15}$. Si la trame est commune à tous ces supports ${ }^{16}$, c'est qu'elle est particulièrement adaptée à un retour réflexif sur l'expérience de travail, ce que l'on va détailler dans l'analyse des usages de l'agenda. Mais avant cela, il faut encore préciser les changements apportés aux techniques de notations, à mesure que l'agriculture se modernise.

\section{Enregistrer, classer les papiers}

Vient une période où l'image du paysan « éclairé », qui tient agenda et fait journellement la balance de ses recettes et dépenses apparaît désuète. Où s'annonce «la fin des paysans ${ }^{17}$, et avec elle, la disparition d'un mode de vie et d'une manière de pratiquer l'agriculture, laissant place à des agriculteurs « entrepreneurs », désireux de s'inscrire dans l'économie moderne et d'en maîtriser le jeu. Ce basculement des années 1950-1960, dépeint comme une véritable révolution mentale, a plusieurs conséquences pour ce qui nous intéresse. Tout d'abord, il conduit à un évincement progressif de l'écriture au profit du calcul, auquel tous les pans de l'activité agricole sont désormais soumis, du lait qui devient « un produit éclaté en divers paramètres qu'il convient de surveiller étroitement ${ }^{18}$ au blé qui doit se cultiver « assis » ${ }^{19}$ pour être produit économiquement, en passant par la calculatoire

14. Marc Dubruel, «Les études agricoles par correspondance », 1928, dans Annales d’histoire des enseignements agricoles, $1992, \mathrm{n}^{\circ} 4-5, \mathrm{p} .74$.

15. Daniel Chartier, A l'aube des formations par alternance, Paris, Editions Universitaires UNMFREO, 1986.

16. Supports utilisés pour l'enseignement, mais on peut supposer que les vulgarisateurs reprennent à leurs comptes ces canevas d'écriture.

17. Henri Mendras, La fin des paysans : vingt ans après, Actes Sud, Paris, 1967 (réed. 1984).

18. Dominique Bourg, "Les agriculteurs, figures de la modernité ", dans Courtet C., BerlanMarque M., Demarne Y., Agriculture et société. Pistes pour la recherche, 1993.

19. M. Artaud, Le métier d'agriculteur et l'agriculture nouvelle, Paris, Les Editions Ouvrières, 1968. 
génétique introduite dans les étables. De plus, la complexité des systèmes productifs devient telle que le recours à des organismes spécialisés se généralise (contrôle laitier, coopérative d'insémination, instituts techniques, centre de gestion...) privant en partie les agriculteurs de leur autonomie de gestion. Enfin, les catégories elles-mêmes avec lesquelles les exploitants peuvent se représenter leur conduite technique et leurs résultats économiques sont imposées de l'extérieur, par la rationalité économique et scientifique. C'est le cas du langage de la comptabilité-gestion qui instaure une mesure chiffrée selon des normes universelles, et ce faisant, établit des lignes de partage entre les exploitants suivant leurs motivations économiques, comme l'ont bien montré les travaux de Placide Rambaud ${ }^{20}$. On peut encore citer l'établissement de l'UGB (Unité de Gros Bétail), mesure qui confère à chaque animal une valeur sur la base de ses besoins alimentaires et qui devient rapidement une norme technico-administrative incontournable.

Dans ce contexte de mutations rapidement brossé, tenir chronique paraît anachronique : « Primitivement, l'agriculteur utilisa l'agenda dans lequel il essayait de porter le maximum de renseignements sur les événements de la ferme. Cette méthode est insuffisante car ne disposant pas d'un guide pour les enregistrements, il commet de nombreuses omissions et la nature des rubriques étant laissée au libre choix de chacun, elles s'avèrent difficilement exploitables ${ }^{21}$. L'enjeu, pour les agents de développement, est d'amener les exploitants à rationaliser leurs méthodes de gestion, en prenant exemple sur le chef d'entreprise ${ }^{22}$. Il s'agit de leur apprendre à raisonner l'efficacité de chacun de leurs facteurs de production, et à ne pas se contenter d'une appréciation d'ensemble des résultats : "Il faut contrôler les résultats, l'ère de l'à-peu-près est révolue ! » ${ }^{23}$. Par différents biais - revues professionnelles, conseil individuel groupes de développement, cercles jacistes ${ }^{24}$, stages de formation -, les agriculteurs s'initient aux " enregistrements », terme qui consacre à présent une écriture réduite à sa plus simple expression, souvent à partir de supports spécialisés. Ils sont également guidés dans la gestion de leur documentation (plan de classement des papiers de la ferme) et l'équipement du «bureau de l'exploitation », lieu d'une activité présentée comme hautement stratégique. Leurs épouses ne sont pas en reste. Ainsi, dans les groupes de vulgarisation qu'elles fréquentent, elles s'emploient à chiffrer, montre en main, le temps consacré à

20. "Les agriculteurs et leurs langages mathématiques ", Cahiers internationaux de Sociologie, vol. LXXXVII, 1989.

21. Propos du directeur de l'Institut de l'Organisation Scientifique du Travail Agricole Institut, créé en 1947 à l'INRA, dans Jean Piel-Desruisseaux, L'organisation du travail en agriculture, Paris, Les Editions d'Organisation, 1963, p. 221.

22. Il s'agit là d'une tendance générale. Les positions des agents de développement à l'égard des exploitants et leur mode d'engagement dans leur activité de conseil sont en fait plus divers et complexes, comme l'a analysé Bruno Lémery, Lectures sociologiques des activités de conseil technique en agriculture. Essai sur les processus de rationalisation, Thèse de doctorat, Lyon 2, 1991.

23. Slogan tiré des dossiers «L'agriculteur chef d'entreprise », Revue de la FNCETA, Etude n 401, 1960.

24. La Jeunesse Agricole Catholique, créée en 1929, mise sur la capacité autodidacte de la paysannerie pour faire face au défi de la modernisation. Par sa pédagogie du "voir-juger-agir », elle s'emploie à faire acquérir à ses militants de base comme aux cadres du mouvement des méthodes de travail, en particulier des techniques d'écriture. 
chacune de leurs activités, de façon à en rationaliser l'organisation. Les qualités d'ordre et de soin qu'elles sont supposées détenir - dispositions ascétiques, rationnelles et calculatrices forgées par l'habitude de gérer la vie du foyer ${ }^{25}$ - en font par ailleurs les cibles privilégiées des stages de formation à la comptabilité et à la gestion qui se mettent en place.

\section{RÉCITS DU TRAVAIL " À SOI "}

Comment ces modelages successifs d'une écriture en relation avec le travail agraire ont-ils été reçus ? Jusqu'où les canevas proposés, dont la trame se resserre au fil du temps, ont-ils configuré les écrits des agriculteurs et leur réflexion sur le travail ? Après avoir considéré les modalités de " la prescription », intéressons-nous maintenant à l'inventivité des pratiques d'écriture, sachant que les individus prêtent souvent "une attention oblique » aux modèles et aux messages culturels, comme l'a bien montré Roger Chartier ${ }^{26}$ au sujet des pratiques de lecture populaires.

De récentes enquêtes menées au sujet de la traçabilité ${ }^{27}$ confirment l'habitude de tenir chronique et sa valorisation dans le milieu professionnel : cela présume d'une exploitation "bien tenue ». Du reste, c'est en prenant au sérieux ces réputations d'écrivants que nous avons pu engager notre recherche sur les écrits du travail au milieu des années $1990^{28}$. Il est intéressant de noter que la tenue d'agenda semble particulièrement répandue chez ceux qui ont pris le train du progrès dans les années 1960 et ont été les premiers à adhérer au centre de gestion, au contrôle laitier et à participer à des groupes de développement, alors même que ce genre d'écriture commençait à être déprécié et que s'amorçait une délégation des enregistrements, comme nous venons de le voir. L'analyse des pratiques de l'écrit que nous présentons est tirée d'une enquête ethnographique menée auprès de huit agriculteurs de Haute-Saône, entre 1995 et 1997. Ils ont été choisis en raison de leur écriture quotidienne et de l'archivage de leurs agendas. Quelquefois, le support était tenu à deux mains, entre mari et femme. Suivant les scripteurs, nous disposions de corpus couvrant quinze à quarante années d'écriture. La plupart, aujourd'hui à la retraite, était à la tête d'exploitations de polyculture-élevage de taille moyenne. Leur pratique d'écriture, souvent enracinée dans une tradition familiale, nous a permis d'accéder à de vieux documents (livres de comptes, carnets de parents et grands-parents).

Que trouve-t-on dans ces supports maniés au quotidien ? En un style fortement stéréotypé, l'écriture égrène jour après jour les occupations des exploitants : travaux des champs, soin aux animaux, bricolages, déplacements... À première vue, ces écrits "à soi » possèdent toutes les caractéristiques des écrits en

25. Suivant l'analyse que propose Bernard Lahire, La raison des plus faibles : rapport au travail, écritures domestiques et lectures en milieux populaires. PUL, Lille, 1993.

26. Roger Chartier, Lectures et lecteurs dans la France d'Ancien Régime, Paris, 1987, p. 13.

27. Nathalie Joly, Jean-Marc Gautier « Pratiques de la traçabilité en élevage : des compétences à ajuster », Actes des $13^{\mathrm{e}}$ Rencontres de Recherche sur les Ruminants, en ligne, 2006.

28. Nathalie Joly, Ecritures du travail et savoirs paysans. Aperçu historique et lecture de pratiques. Les agendas des agriculteurs, thèse en Sciences de l'Education, Paris-X Nanterre, 1997. 
organisation $^{29}$, restituant la pratique avec une économie du système linguistique et sémiotique et un effacement des scripteurs. Comme n'importe quel agent chargé de relater son activité au sein d'un service ou d'un atelier, l'agriculteur rapporte ses interventions (parfois celle de ses proches) à l'aide de formules répétitives, souvent sans conjugaison, avec des rares emplois du « je ». La description du travail peut être plus ou moins détaillée : les uns récapitulent leur journée en quelques mots, d'autres rapportent les aspects techniques de leurs interventions (quantité d'engrais utilisés, réglages du matériel, hectares travaillés), d'autres encore complètent ce compte rendu d'appréciations (effet de la météo sur les cultures, avantage d'une procédure). Parfois, un lieu est si évidemment lié à une activité et à un moment de la journée qu'il les désigne tout à la fois, prenant valeur de « chronotope ${ }^{30}$. Systématiquement, le texte se découpe en séquences d'activité, séparées d'un trait à la main ou d'une ponctuation qui marquent les " pores » 31 de la journée de travail. L'agenda restitue donc la chronologie des activités, et pour les plus soucieux de précision et d'analyse, la réflexion qui l'accompagne, comme ci-dessous :

"Ce matin conduire ma voiture de fumier / Descendre de la graine de la moisson du dessus blé et Orge / Laver mes fenêtres d'écurie / Après-midi aller à Vesoul faire des courses rentrer à 5 heures / Ce soir après la traite aller faire le tour du parc à Chêne Béni et ouvrir la parcelle neuve » (Roger).

«Semé maïs Crop autour du Hangar LG 11 / Semé avec 650. 1800 tours 4e petite. Traceur sortir de 4,5 cm $1 \mathrm{~m} 20$ entre la roue tracteur et le disque traceur ", " Foin grands Prés. Temps frais pour la saison. 913 bottes Total. Le veau de la Noire Michotte est crevé (gros nombril). Aider Fernand Joly» (Albert).

« Temps nuageux à beau, très chaud, encore orageux l'après-midi $\left(+12^{\circ}+28,5^{\circ}\right)$. Traité les jardins contre les insectes et les maladies » (après les pluies d'orages)

Maïs : pousse bien après la pluie. 6 à 8 feuilles (il a grêlé dimanche soir à la combe. Brouillard - feuilles lacérées) (et aussi au Fourbier un peu moins)

Soja : $2-3^{\mathrm{e}}$ nœud - pousse bien

Tournesol : bonne pousse aussi. Monte 20 à $40 \mathrm{~cm}$ de haut - 5-7 rangs de paires de feuilles (bientôt application de Bore)

O-H : Formation du grain - début de jaunissement des barbes (un peu d'helmétho - habituel -)

Blé : Floraison - Fin d'épiaison (sauf forby - épié à 70 \%)

Colza : grossissement des siliques (il y a comme souvent des siliques perdues en bordure par le charançon des siliques)

O.P. : épiaison débutante (pas de verse après les orages) (René).

29. Pour une rétrospective de ces recherches, se reporter à l'ouvrage dirigé par Anni Borzeix et Béatrice Fraenkel, Langage et travail. Communication, cognition, action, CNRS Editions, Paris, 2001.

30. Philippe Lucas emprunte cette notion à Mikhail Bakhtine (Esthétique et théorie du roman, Paris, Gallimard, 1978) pour rendre compte des corrélations entre le temps et l'espace qui caractérisent les récits de vie quotidienne d'ouvriers mineurs, dans « Les travaux et les jours », Cahiers internationaux de Sociologie, vol. LXXIV, 1983.

31. Expression employée par Karl Marx pour décrire les différentes coupures dans le travail de l'artisan, celui-ci devant changer régulièrement de place ou d'instruments pour réaliser son œuvre. 
Complétant le récit du travail, quelques chiffres renseignent sur la gestion quotidienne (achats d'engrais, vente d'animaux). Dans les pages de fin de mois et de fin d'année qui invitent formellement au bilan, des inventaires apparaissent (stocks de fourrage, état du cheptel) ainsi que diverses listes prévisionnelles (planning de fumures, lots d'animaux pour la reproduction ou la vente). Des opérations griffonnées dans les marges appuient cette activité récapitulative.

"Vendu un veau à Soyer 102 kilos à 475 francs », «Prix du lait année 1963: 42,50 $+3 \mathrm{~F}$ de ristourne $-1 \mathrm{~F}$ capital social ", "Voir contrôle laitier. Avec la mesure économique, coûterait environ 2000 francs, avec $33 \%$ en testage » (Charles).

«Possibilité en pâture 1976 : Vaches Michel Parc (18) Étang (10), Belot (15). Y compris les génisses prêtées nous aurons 86 vaches - je dois donc en vendre $86-60=26$ ", « Nous avons nourri 35 babys moyens avec la dernière travée du silo pendant 60 jours soit 1 ha, 5 de Maïs pour 2100 jours de nourriture de baby soit 1600 jours de Baby/ha soit environ $320000=200 \mathrm{~K} /$ jour » (Marcel).

$\mathrm{Au}$ long de ces chroniques monotones, le lecteur attentif décèle cependant des manières très personnelles de broder autour du récit du travail, d'en élargir ou resserrer l'horizon, suivant la disponibilité ou le goût d'écrire, les événements remarquables de la journée, et peut-être l'humeur des scripteurs. Les incursions dans la vie familiale, ordonnée autour des activités de l'exploitation, sont fréquentes : plusieurs générations s'épaulent au quotidien dont l'écriture témoigne, en signalant l'aide du père ou du fils, la mobilisation de toute la maisonnée en cas d'urgence comme finir les foins avant l'orage. Par une sorte d'effet d'entraînement, l'agriculteur inscrit les faits marquants touchant à la famille (naissances, mariages, décès, travaux d'aménagement du domicile...). On trouve également dans l'agenda tout l'écheveau des relations professionnelles et sociales tissées par l'activité agricole : qu'ils recourent à de l'entraide avec le voisinage et les exploitants doublent l'accord oral d'une trace écrite, faisant le compte de ce qui est donné et reçu, coups de main, prêt de matériel, covoiturage ; qu'ils reçoivent une visite sur l'exploitation, celle du marchand à bestiaux, du vétérinaire ou du conseiller, ou qu'ils se rendent à l'extérieur, à une réunion syndicale ou à la foire locale, le récit du jour le mentionne. Enfin, quelques minces traces de subjectivité affleurent des chroniques, à travers le signalement d'une maladie, d'une contrariété.

«Ce matin avec les Conseillers aller désherber le cimetière terminer à $10 \mathrm{~h} 30$ / Ensuite aller à Goncourt au vétérinaire chercher pour soigner un veau / Après-midi désherber mes orges d'hiver et commencer les blés. Faire Chanceaux - Ch. René Creux Mercier La Vigne Ch de Fécamps Marcel Prudhon Les Combes (contre parc) Les Audières CH Mauvais / Ce soir aller à la confession avec Mathilde à 21 heures » (Roger).

« Manger chez les Munier. Passé un bon moment » (Albert). «Opération du papa à Vesoul » (Roland). « Je suis allée seule à la messe avec Didier. L'après-midi, on est allé aux jonquilles avec notre "petit » chien » (Marie, notant dans les agendas de Charles) 
Ces infimes manifestations « des autres et de soi » donnent chair aux chroniques de l'agenda. Si celles-ci se construisent bien autour des opérations de travail, en droite ligne des prescriptions exposées précédemment, elles renseignent également sur les micro-univers des scripteurs. Tenir agenda, ce n'est pas seulement se constituer une réserve de faits sur le travail, se fabriquer une " mémoire grenier ${ }^{32}$ pour reprendre la formule suggestive de Christian Bromberger et Anne-Hélène Dufour. C'est aussi une façon de garder trace d'une vie. Les plus soucieux de perfectionnement technique ou de calculs économiques expriment leur attachement à cette dimension de l'agenda. La mémoire utilitaire devient mémoire familiale: "C'est un peu toute notre vie qu'il y a là-dedans ", nous confie l'un des scripteurs. Ainsi, il n'est pas rare qu'un vieux support soit consulté lors de retrouvailles, ravivant un souvenir lointain. Des enjeux identitaires croisent donc les enjeux de connaissance qui justifient initialement la prise d'écriture. On comprend mieux dès lors certaines résistances face aux outils d'enregistrement, la difficulté à adopter des " prêt-àécrire ${ }^{33}$ confectionnés suivant une logique strictement utilitaire.

\section{RESSOURCES POUR L'ACTION}

Agir sur le vivant, végétal ou animal, c’est savoir décider en situation. Prélever des indices jamais donnés une fois pour toutes. Cette capacité essentielle des travailleurs du vivant à se forger des repères spatio-temporels, les paludiers la ramassent en une formule : "On ne peut rien prévoir, mais on se souvient ${ }^{34}$. Tenir agenda, c'est se donner les moyens de se souvenir et de rapporter les événements du travail à l'échelle objective d'un calendrier, ce qui engage une maîtrise profondément novatrice du temps, en rupture avec la connaissance traditionnelle et routinière. Pédagogues et vulgarisateurs ont bien perçu l'intérêt d'une écriture journalière, suivie de synthèses, car elle autorise une prise de distance vis-à-vis du sens pratique et un retour réflexif sur les actes de travail. Les agriculteurs jouent-ils avec ces mécanismes de la « raison graphique ${ }^{35}$ et de quelle façon?

C’est généralement le matin, au petit-déjeuner, que l'agenda est consulté. On en feuillette les pages pour se représenter l'avancée d'un chantier qui peut s'étaler sur plusieurs jours comme plusieurs semaines. La chronique du travail permet de tenir le fil de l'activité ${ }^{36}$, de réfléchir à « ce qui est fait et reste à faire », puis de fixer le programme des jours suivants. La capacité de l'agenda à rappeler le séquençage

32. « Du bon usage du temps. L'agenda agricole d'un ouvrier-varois en 1942 », Provence Historique, tome XXXIII, fasc. 132,1983 .

33. L'expression est de Sonia Branca-Rosoff, «Conventions d'écriture dans la correspondance ses soldats », Mots, $\mathrm{n}^{\circ} 24,1990$.

34. Geneviève Delbos, « Savoir du sel, sel du savoir », Terrain, ${ }^{\circ} 1,1983$, p. 18.

35. Selon la fameuse formule de l'anthropologue Jack Goody qui a bien montré comment l'écriture permettait le réexamen ou le réagencement des données perceptives et souligné, en particulier, les effets cognitifs de certains procédés graphiques comme la mise en liste ou la mise en tableau, dans La raison graphique, Paris, Editions de Minuit, 1979.

36. Nathalie Joly, «Ecrire l'événement. Le travail agricole mis en mémoire, Sociologie du travail, vol. 46, $\mathrm{n}^{\circ} 4,2004$. 
des interventions (dates et parcelles travaillées) est essentielle, car l'activité des exploitants est particulièrement dispersée dans le temps et l'espace. Le support contribue ainsi à la maîtrise des rapports temporels entre les événements du travail, sur une base autre que celle de l'expérience, des dictons, ou encore, des coutumes locales. Mais deux conditions sont à réunir pour cela : il faut que l'écriture soit régulière et que toute occupation figure, les tâches nobles comme les moins nobles (le nettoyage et le bricolage) ou encore les «à-côtés » du métier (réunions professionnelles, visites de tiers), de façon à ce que les exploitants disposent d'un calendrier réel du travail, d'un panoptique de leur activité. Pour naviguer avec aisance à l'intérieur de ce calendrier, tous signalent le commencement et l'achèvement des opérations-clés (labours, semis, fertilisation et récoltes pour les cultures ; mise aux parcs, retour à l'étable et interventions critiques pour le troupeau) et prennent soin de mettre en relief ces butées temporelles (informations cochées ou soulignées).

Le réglage des aspects les plus fins de l'activité, en particulier de ses modes opératoires, nécessite la recherche d'autres informations qui figurent, celles-là, dans l'agenda de la « campagne » précédente. On peut distinguer trois formes de traitement des contenus de l'agenda jouant le rôle de témoin d'un cycle achevé. Dans un premier cas, le plus fréquent, les agriculteurs se contentent d'une simple reprise des procédures décrites qu'ils adaptent à la situation du moment, notamment à l'état du sol et aux conditions météorologiques : "Avant de commencer le travail, je consulte mes livres de bord. J'ai au moins un an et demi de programmes anciens qui ont été appliqués dans les champs et que je consulte avant d'établir celui de la journée. Par exemple tel produit, telle molécule sont arrivés sur le marché, j’ai utilisé tel produit à telle dose. Bon, je prends ma calculette et je fais mon programme. Et puis, hop, dans la poche de la cotte, le programme nouveau et celui de l'année d'avant, ça se joue au $\mathrm{cm}^{3}$ près » (René).

Dans un second cas, ils effectuent des comparaisons de "campagne » à « campagne » qui prennent en compte plusieurs variables (différentes dates d'intervention, des données météorologiques). Cette combinatoire complexe soutient alors l'évaluation d'une activité, l'interprétation d'une situation. Les plus perfectionnistes prennent au-delà de deux années de référence : "C’était le 17 avril qu'on a semé et puis, on a récolté le 25 septembre, l'année encore avant, c'était le 29 mars et les récoltes le 31 août. Et voyons voir l'année encore avant ?, alors c'était comme ça que je me faisais une idée » (Charles).

Dans un troisième cas, les scripteurs retournent à l'agenda de l'année précédente pour compléter, et quelquefois corriger, certaines données inscrites. Cela permet de codifier progressivement les résultats de l'expérience, particulièrement des tours de main qui occupent les pages dévolues aux notes de fin d'année : comment alimenter une vache tarie, fabriquer des cordes à foin, soigner une bête. Ceux qui se risquent à des essais sur quelques ares de terre parviennent à dégager des règles d'action, à l'aide de ce processus itératif d'écriture/observation/ réécriture, comme ci-dessous :

"Semaille blé (1960) : Aux Saves, semé à sec au 3 - peut être un peu faible, graine belle, milieu au 3,1 " - voir différence, " Dormand, champ de Marcel, semé Trèfle violet + Rina 5, 1/2. Voir levée. (Ajout) : Mettre 6 sans inconvénient ». «Plantation 
betteraves 1969 : Planter le 5 mai 1969 : Labourer - passer le rotovator deux fois, une fois en $2^{\mathrm{e}}$ et l'autre en troisième petite - semer engrais complet et sulfate d'ammoniaque - herser - rouler - planter - ne pas rouler derrière c'est inutile. En temps de pluie désherber le soir de préférence et dans les cinq jours qui suivent le semis » (Albert).

Si l'agenda correspond bien à un instrument $\operatorname{cognitif}^{37}$, qui vient soutenir la réflexion des agriculteurs à des phases intenses de diagnostic, il est également mobilisé à des fins de routinisation de l'activité, rôle plus imprévu joué par le support. Particulièrement à propos des cultures, car en ce domaine, la mémoire de l'exploitant est très sollicitée, du fait de nombreuses interactions entre variables au cours du cycle de production, du caractère en grande partie incertain et fréquemment différé dans le temps des résultats obtenus et de l'action aléatoire du climat. Bien que les exploitants connaissent théoriquement les dates auxquelles il leur faut labourer, semer, récolter, ils savent également que « chaque opération du cycle possède un type de temporalité spécifique et demande une configuration précise du temps (météorologique) à des moments de l'année ${ }^{38}$. Or, les collections d'agendas fabriquent une mémoire du travail qui croise ces deux coordonnées, le temps calendaire et le temps météorologique. Le calendrier du cycle précédent s'offre alors comme un " calendrier de routine ${ }^{39}$ qui nourrit des « attentes d'événements » à partir desquelles " réduire l'incertitude et la complexité des situations de décision et d'action ${ }^{40}$. Les informations sont en effet " arrangées » de telle sorte que les exploitants peuvent aisément estimer l'avancée et le retard pris dans leurs interventions et en comprendre les raisons : un hiver rigoureux a retardé les semis de printemps, les chaleurs de Mai annoncent une fenaison précoce. Cette possibilité de jugement est essentielle aux exploitants, essentielle aux exploitants, anxieux de pouvoir "faire à temps " ${ }^{41}$ et pour lesquels ce dispositif d'écriture est une sorte de " réassurance mentale contre la menace de l'aléa » ${ }^{42}$. Lorsque d'importants écarts à la normale se produisent, ils sont dès lors interprétables et rationalisables : «Un des impondérables du métier, ce que l'on ne maîtrise pas, c'est le temps, mais on peut quand même se l'apprivoiser dans un cursus intellectuel » (René).

37. Pierre Rabardel, Les hommes et les techniques. Approche cognitive des instruments contemporains. Armand Colin, 1995.

38. Lucien Demonio, "La quadrature du cycle. Logique et contraintes du temps en milieu rural », Cahiers Internationaux de Sociologie, $\mathrm{n}^{\circ}$ 67, 1979.

39. Au sens où les exploitants peuvent faire l'économie de représentations internes en prenant appui sur des indices stabilisés contenus dans l'agenda, Nathalie Joly, op. cit., 2004.

40. Louis Quéré, 1997, «La situation toujours négligée? », Réseaux, n 85, p. 171.

41. Angoisse caractéristique des travailleurs du vivant que décrit Michèle Salmona dans Le gai savoir des jardiniers : le vivant/animalier et la transmission des savoirs et savoir faire : algorithme/mimésis/phobie, Paris, Centre d'anthropologie économique et sociale, 1983 ainsi que Geneviève Delbos, dans " Les paludiers de Guérande et la météo », Ethnologie Française, 2 (3) 1982.

42. Rôle habituellement joué par les dictons météorologiques, Martin de la Soudière, "Revisiter la météo ». Etudes rurales, n $118-119,1990$. 


\section{De nouveaux Questionnements}

L'exigence actuelle d'une transparence des modes de production, visant à garantir sécurité alimentaire, qualité des produits et respect de l'environnement, s'accompagne de nouvelles exigences rédactionnelles et de gestion documentaire de la part des exploitants. Une évolution vécue comme une mise en cause de l'identité professionnelle des producteurs, de leur conception du métier et de sa condition d'exercice, en "relative » indépendance ${ }^{43}$. Évolution qui suscite par ailleurs quantité d'initiatives, parfois désordonnées, de l'appareil de développement, afin de mettre au point des outils de traçabilité simples, efficaces, informatisés, interopérables... À quoi sert-il dès lors, de rappeler que les tâches d'écriture et de gestion de papiers ne sont pas entièrement nouvelles dans les exploitations? D'insister sur le fait que nombre d'agriculteurs tiennent agendas aujourd'hui et que des usages anciens se superposent aux usages de la modernité, à l'utilisation de logiciels d'aide à la décision et de la technologie internet ? De s'intéresser à des chroniques du travail qui semblent dater d'un autre âge ? Nous pensons qu'un tel détour permet de dégager des questionnements sur la portée des changements en cours et d'y apporter quelques éclairages, suivant trois directions principales.

Premièrement, une telle approche permet de mettre en évidence les compétences développées au travers des multiples actes de scription qui entourent le travail agricole et des activités de gestion d'information : celles mobilisées dans les " écrits pour soi », notations techniques ou économiques et celles attachées à une écriture administrative qui réclame de " cocher, griser, noircir case après case ${ }^{44}$, que les femmes ont prise en charge, de longue date, dans beaucoup d'exploitations ; celles associées au maniement de supports papiers et celles acquises dans l'utilisation de l'informatique; celles encore relatives à l'archivage et au classement de documents. Mais elle invite aussi à s'interroger sur les conditions de leur mise à jour. Les capacités en jeu dans cet ensemble hétérogène d'activités se transfèrent-elles d'une situation à une autre ? Comment, en particulier, des écrits « pour soi » s'agencent-ils à des écrits destinés à des tiers ? Nous avons vu l'attention portée, dans la tenue d'agenda, aux dates, localisations et procédures d'interventions. Ces informations sont désormais réclamées au titre de la réglementation et d'engagements contractuels, souvent à partir de catégories de description qui diffèrent des outils indigènes : faut-il que les exploitants deviennent bilingues ou doivent-ils simplement se plier à quelques ajustements de langage, autorisant le passage d'un monde à l'autre, de la pratique professionnelle au domaine du réglementaire et de la traçabilité ? Quelles sont, en définitive, les particularités du «travail administratif », notion qui fait couler beaucoup d'encre mais dont les contours sont mal circonscrits ${ }^{45}$ ?

Deuxièmement, notre détour par les pratiques ordinaires de l'écrit, en regard des prescriptions dont elles ont fait l'objet, offre un éclairage sur les modes d'appropriation des techniques de l'écrire-compter-mesurer et les dynamiques

43. Au regard des formes d'intégration de l'agriculture à des complexes agro-industriels.

44. Claudine Dardy, Objets écrits et graphiques à identifier, Paris, L’Harmattan, 2004.

45. Le terme a surtout pour effet de déclencher les protestations de la profession agricole qui s'inquiète de l'importance donnée aux aspects réglementaires et aux contrôles. 
collectives dont ils relèvent. En sera-t-il de même dans le processus actuel de normalisation de l'activité agricole? Pour l'instant, les exploitants semblent plus sujets qu'acteurs de la conception des dispositifs de traçabilité, mais les capacités dont ils ont fait preuve par le passé pour aménager les modèles et les reformuler selon leurs propres perspectives, invitent à laisser ouvert le questionnement et à être attentif aux formes d'inventivité qui accompagnent leur mise en œuvre.

Troisièmement, s'intéresser au support agenda et à ses usages quotidiens, c'est mieux cerner l'importance de cette « littérature grise du travail » ${ }^{46}$, les divers appuis qu'elle procure aux scripteurs dans la conduite de leur activité. Mais qu'adviendrat-il de toute cette literacy dans un univers gouverné par l'informatique, qui affiche avec assurance l'objectif du "zéro papier " ? Ce questionnement mérite d'être soulevé, non par nostalgie, mais par souci de rappeler la vertu réflexive des actes d'écriture - inscriptions, recopiages, brouillons - que les manuels jacistes d'avantguerre résumaient en une formule imagée : «Mâchez, disait le docteur de famille quand nous étions enfants, la nourriture vous profitera deux fois plus. Prendre des notes équivaut à cette mastication intellectuelle ${ }^{47}$ ».

\section{NATHALIE JOLY}

46. Sophie Pène «Analyse de postes, bilans d'entretien, écriture de procédures. Un prêt-à-écrire pour inscrire l'activité ", Education Permanente, n³, 1994.

47. Maurice Gaudillière, Eléments d'une culture paysanne. Divione, Collection Semailles, 1942. 